\title{
A CHARACTERIZATION OF A CLASS OF BARRELLED SEQUENCE SPACES
}

\author{
by J. SWETITS
}

(Received 19 May, 1976)

1. Introduction. In a recent paper [4] Bennett and Kalton characterized dense, barrelled subspaces of an arbitrary $F K$ space, $E$. In this note, it is shown that if $E$ is assumed to be an $A K$ space, then the characterization assumes a simpler and more explicit form.

2. Definition and preliminaries. $\omega$ denotes the vector space of sequences of complex numbers. A subspace $E$ of $\omega$ is a $K$ space if it is endowed with a locally convex topology $\tau$ such that the linear functionals

$$
x \rightarrow x_{i} \quad(j=0,1,2, \ldots)
$$

are continuous. In addition, if $\tau$ is complete and metrizable, then $(E, \tau)$ is an $F K$ space.

If $x=\left\{x_{k}\right\}$, let $P_{n} x=\left\{x_{0}, x_{1}, \ldots, x_{n}, 0, \ldots\right\}$. If a $K$ space $(E, \tau)$ has the property that $P_{n} x \rightarrow x$ in $\tau$ for each $x \in E$, then $(E, \tau)$ is called an $A K$ space.

If $E$ is an $F K-A K$ space then the dual of $E$ may be identified with

$$
E^{\beta}=\left\{y \in \omega: \sum_{i=0}^{\infty} x_{i} y_{i} \text { converges } \forall x \in E\right\} .
$$

If $F$ is a subspace of $E^{\beta}$ containing the space $\phi$ of sequences with only finitely many non-zero terms then $E, F$ form a separated pair under the bilinear form

$$
\langle x, y\rangle=\sum_{i=0}^{\infty} x_{i} y_{j} .
$$

$\sigma(E, F), \tau(E, F)$ and $\beta(E, F)$ denote the weak, Mackey and strong topologies, respectively, on $E$ by $F$ (see, e.g., [7]).

If $A=\left(a_{n k}\right)$ is an infinite matrix of complex numbers, the sequence $A x=\left\{(A x)_{n}\right\}$ is defined by

$$
(A x)_{n}=\sum_{k=0}^{\infty} a_{n k} x_{k} \quad(n=0,1,2, \ldots) .
$$

$E_{\mathrm{A}}=\{x: A x \in E\}$, where $E$ is a given sequence space. $A^{\prime}$ denotes the transpose of $A$.

The following theorem is established in [8].

THEOREM 2.1. Let $E$ and $F$ be sequence spaces, each containing $\phi$, such that $\left(E^{\beta}, \sigma\left(E^{\beta}, E\right)\right)$ and $\left(F, \sigma\left(F, F^{\beta}\right)\right)$ are sequentially complete. If $A=\left(a_{n k}\right)$ is an infinite

Glasgow Math. J. 19 (1978) 27-31 
matrix, then the following are equivalent:

(i) $F_{A}$ contains $E$;

(ii) $E_{A^{\prime}}^{\beta}$ contains $F^{\beta}$;

(iii) $F_{A^{\prime}}$ contains $\left(E^{\beta}\right)^{\beta}$.

Proof. (i) $\Rightarrow$ (ii). Let $\left\{t_{k}\right\} \in F^{\beta}$ and $\left\{x_{k}\right\} \in E$. Define the matrix $B=\left(b_{n k}\right)$ by

$$
b_{n k}= \begin{cases}t_{k} & (0 \leq k \leq n) \\ 0 & (k>n)\end{cases}
$$

Then

$$
\begin{aligned}
\sum_{n=0}^{\infty} t_{n} \sum_{k=0}^{\infty} a_{n k} x_{k} & =\lim _{j \rightarrow \infty} \sum_{n=0}^{j} t_{n} \sum_{k=0}^{\infty} a_{n k} x_{k} \\
& =\lim _{j \rightarrow \infty} \sum_{k=0}^{\infty} x_{k} \sum_{n=0}^{j} t_{n} a_{n k} \\
& =\lim _{j \rightarrow \infty}[(B A) x] j .
\end{aligned}
$$

The hypotheses on $E$ insure that

$$
\begin{aligned}
\lim _{j \rightarrow \infty}[(B A) x]_{j} & =\sum_{k=0}^{\infty} x_{k} \lim _{j \rightarrow \infty}\left[(B A) e^{k}\right]_{j} \\
& =\sum_{k=0}^{\infty} x_{k} \sum_{n=0}^{\infty} t_{n} a_{n k},
\end{aligned}
$$

where $e^{k}$ denotes the sequence with a one in the $k$ th coordinate and zeros elsewhere.

Since $\left\{t_{k}\right\} \in F^{\beta},\left\{x_{k}\right\} \in E$ are arbitrary, it follows that $A^{\prime}$ maps $F^{\beta}$ to $E^{\beta}$.

(ii) $\Rightarrow$ (iii) follows from (i) $\Rightarrow$ (ii) and the fact that $F=\left(F^{\beta}\right)^{\beta}$ if $\left(F, \sigma\left(F, F^{\beta}\right)\right)$ is sequentially complete $[10$, p. 974$]$.

(iii) $\Rightarrow$ (i) is trivial.

\section{A class of barrelled spaces.}

THEOREM 3.1. Let $E$ be an FK-AK space and $E_{0}$ a subspace of $E$ containing $\phi . E_{0}$ is barrelled in $E$ if and only if

(i) $E_{0}^{\beta}=E^{\beta}$, and

(ii) $\left(E^{\beta}, \sigma\left(E^{\beta}, E_{0}\right)\right)$ is sequentially complete.

Proof. (Necessity) Let $\left\{t_{k}\right\} \in E_{0}^{\beta}$, and define $A=\left(a_{n k}\right)$ by

$$
a_{n k}= \begin{cases}t_{k} & (0 \leq k \leq n), \\ 0 & (k>n) .\end{cases}
$$


If $c$ denotes the space of convergent sequences, then $c_{\mathrm{A}}$ includes $E_{0}$. Since $c_{\mathrm{A}}$ is an $F K$ space [9, ch. 12], it follows from [4, Theorem 1] that $c_{A}$ includes $E$. Thus, for any $x \in E, \sum_{k=0}^{\infty} t_{k} x_{k}$ converges. Consequently $E^{\beta}$ includes $E_{0}^{\beta}$. Since the reverse inclusion is satisfied, we have $E_{0}^{\beta}=E^{\beta}$.

Let $\left\{a^{(n)}\right\}$ be a sequence in $E^{\beta}$ that is $\sigma\left(E^{\beta}, E_{0}\right)$ Cauchy. If $A=\left(a_{n k}\right)$ is defined by $a_{n k}=a_{k}^{(n)}$, then $c_{\mathrm{A}}$ includes $E_{0}$. Consequently, $c_{\mathrm{A}}$ includes $E$, [4, Theorem 1]. Condition (ii) now follows from the the fact that $E^{\beta}$ is $\sigma\left(E^{\beta}, E\right)$ sequentially complete.

(Sufficiency). Let $\left\{a^{(n)}\right\}$ be a sequence in $E^{\beta}$ that is $\sigma\left(E^{\beta}, E_{0}\right)$ bounded. Let $m$ denote the space of bounded sequences, and define $A=\left(a_{n k}\right)$ by $a_{n k}=a_{k}^{(n)}$. Then $m_{A}$ includes $E_{0}$. Conditions (i) and (ii) and Theorem 2.1 imply that $m_{A}$ includes $E$ since $(m, \sigma(m, \ell))$ is sequentially complete $\left(\ell=\right.$ space of absolutely convergent series). Thus, $\sigma\left(E^{\beta}, E_{0}\right)$ and $\sigma\left(E^{\beta}, E\right)$ define the same bounded sequences and, hence, the same bounded sets. Thus, the topology $\beta\left(E_{0}, E^{\beta}\right)$ is the restriction of $\beta\left(E, E^{\beta}\right)=\tau\left(E, E^{\beta}\right)=F K$ topology of $E$ to $E_{0}$. It follows that $E_{0}$ is barrelled in $E$.

Remarks. If $E_{0}$ is monotone (i.e., the coordinatewise product $x y \in E_{0}$ if $x \in E_{0}$ and $y$ is a sequence of zeros and ones) then condition (ii) of Theorem 3.1 can be omitted [3,p. 55].

Let $\left\{r_{n}\right\}$ denote a non-decreasing unbounded sequence of positive integers with $r_{0}=1$ and $r_{n}=o(n)$. For each $x \in \omega$ and each $n=0,1,2, \ldots$, let $c_{n}(x)$ denote the number of non-zero elements in $\left\{x_{0}, x_{1}, \ldots, x_{n}\right\}$. If $E$ is a sequence space, a scarce copy of $E$ is the linear span of

$$
\left\{x \in E: c_{n}(x) \leq r_{n}, n=0,1,2, \ldots\right\} \text {. }
$$

As corollaries to Theorem 3.1, we obtain Theorems 7, 8 and 10 of [2]. In each case the spaces are monotone and the verification of condition (i) of Theorem 3.1 is straightforward.

$\omega$ has the topology of coordinatewise convergence, and, for $p>0, \ell^{p}=$ $\left\{x: \sum_{j=0}^{\infty}\left|x_{j}\right|^{p}<\infty\right\}$.

COROLLARY 3.2. Every scarce copy of $\omega$ is barrelled.

COROLLARY 3.3. Every scarce copy of $\bigcap_{p>0} \ell^{p}$ is barrelled as a subspace of $\ell$.

COROLlary 3.4. Let $E$ be a monotone FK-AK space. The union of all the scarce copies of $E$ is a barrelled subspace of $E$.

It is noted that Corollary 3,4 strengthens Theorem 10 of [2], which is stated for solid spaces.

Another consequence of Theorem 3.1 is the following result. 
COROLlary 3.5. Let $E$ be an FK-AK space and $E_{0}$ a subspace of $E$ containing $\phi$. The following are equivalent:

(i) $E_{0}$ is barrelled;

(ii) If $G$ is a separable $F K$ space containing $E_{0}$, then $G$ contains $E$.

Proof. (i) $\Rightarrow$ (ii). This is a consequence of $\left[4\right.$, Theorem 1]. (ii) $\Rightarrow$ (i). Let $\left\{t_{k}\right\} \in E_{0}^{\beta}$, and define $A=\left(a_{n k}\right)$ by

$$
a_{n k}= \begin{cases}t_{k} & (0 \leq k \leq n), \\ 0 & (k>n) .\end{cases}
$$

Then $c_{\mathrm{A}}$ includes $E_{0}$. Since $c_{\mathrm{A}}$ is a separable $F K$ space [1, p. 199], $c_{\mathrm{A}}$ includes $E$. Thus, $\left\{t_{k}\right\} \in E^{\beta}$, and condition (i) of Theorem 3.1 is satisfied.

Let $\left\{a^{(n)}\right\}$ be a sequence in $E^{\beta}$ that is $\sigma\left(E^{\beta}, E_{0}\right)$ Cauchy. If $A=\left(a_{n k}\right)$ is the matrix defined by $a_{n k}=a_{k}^{(n)}$, then $c_{\mathrm{A}}$ includes $E_{0}$. It follows that $c_{\mathrm{A}}$ includes $E$. Since $E^{\beta}$ is $\sigma\left(E^{\beta}, E\right)$ sequentially complete, condition (ii) of Theorem 3.1 is satisfied. Thus, $E_{0}$ is barrelled.

Remark. For $F K-A K$ spaces, (ii) $\Rightarrow$ (i) of Corollary 3.5 improves (ii) $\Rightarrow$ (i) of $[4$, Theorem 1].

In Theorem 3.1, if it is not assumed that $E$ is an $A K$ space, then (i) and (ii) are not sufficient to insure that $E_{0}$ is barrelled in $E$.

Let $E$ be $a c_{0}$, the space of sequences that are almost convergent to 0, (see [6]). For $x \in a c_{0}$, let

$$
\|x\|=\sup _{n}\left|x_{n}\right|
$$

Let $E_{0}=b s+c_{0}$, where

$$
\begin{gathered}
c_{0}=\left\{x \in \omega: \lim _{n \rightarrow \infty} x_{n}=0\right\}, \\
b s=\left\{x \in \omega: \sup _{n}\left|\sum_{j=0}^{n} x_{i}\right|<\infty\right\} .
\end{gathered}
$$

Then $E_{0}^{\beta}=E^{\beta}=\ell$, and $E_{0}$ is dense in $E$ [5, p. 29]. Furthermore, $\ell$ is $\sigma\left(\ell, E_{0}\right)$ sequentially complete. However, $E_{0}$ is a normed $F K$ space when topologized by

$$
\|x\|=\inf \left\{\sup _{n}\left|y_{n}\right|+\sup _{n}\left|\sum_{j=0}^{n} z_{j}\right|: x=y+z, y \in c_{0}, z \in b s\right\} .
$$

It follows from [4, Theorem 1] that $E_{0}$ is not barrelled in $E$.

\section{REFERENCES}

1. G. Bennett, A representation theorem for summability domains, Proc. London Math. Soc. 24 (1972), 193-203. 

$17-30$.

2. G. Bennett, Some inclusion theorems for sequence spaces, Pacific J. Math. 46 (1973),

3. G. Bennett, A new class of sequence spaces with applications in summability theory, $J$. Reine Angew. Math. 266 (1974), 49-75. 511-524.

4. G. Bennett and N. J. Kalton, Inclusion theorems for K spaces, Canad. J. Math. 25 (1973),

5. G. Bennett and N. J. Kalton, Consistency theorems for almost convergence, Trans. Amer. Math. Soc. 198 (1974), 23-43.

6. G. G. Lorentz, A contribution to the theory of divergent sequences, Acta. Math. 80 (1948), 167-190.

7. A. P. Robertson and W. J. Robertson, Topological vector spaces, (Cambridge, 1963).

8. J. Swetits, On the relationship between a summability matrix and its transpose, Old Dominion University, Department of Mathematical and Computing Sciences Technical Report, TR76-2, (1976).

9. A. Wilansky, Functional analysis (Blaisdell, New York, 1964).

10. D. J. H. Garling, The $\beta$ and $\gamma$ duality of sequence spaces, Proc. Cambridge Philos. Soc. 63 (1967), 963-981.

Department of Mathematical and Computing Sciences

OLd DOMINION University

NORFOLK, VIRGINIA 23508 